\title{
El Control Interno en las Empresas \\ PRIVAdas
}

\author{
Mg. Jeri Gloria Ramón Ruffner \\ Docente Asociada
}

\begin{abstract}
RESUMEN
El control interno de las empresas privadas se ha convertido últimamente en uno de los pilares en las organizaciones empresariales, pues nos permite observar con claridad la eficiencia y la eficacia de las operaciones, y la confiabililidad de los registros y el cumplimiento de las leyes, normas y regulaciones aplicables.

El control interno se sustenta en la independecia entre las unidades operativas, en el reconocimiento efectivo de la necesidad de contar con un control interno y la fijación de responsabilidades. Sólo as podrá tener éxito.

En consecuencia podríamos decir que el control interno es de vital importancia, ya que promueve la eficiencia y asegura la efectividad y, sobre todo, previene que se violen las normas y los principios contables.
\end{abstract}

Palabras clave: Evaluación, Independencia, eficiencia y eficacia, información financiera, responsabilldad, control, ética.

\section{INTRODUCCION}

La preocupación que existe sobre las empresas privadas es el manejo del control interno, últimamente se ha podido ver gran cantidad de grandes y medianas empresas que de la noche a la mañana ya no lo eran. Algunas empresas quebradas, otras en reestructuración y otras declaradas como insolventes. Frente a estos hechos las interrogantes que nos planteamos es si acaso los hechos mencionados no son previsibles, si los estados financieros no son un parámetro de medición o es que el patrimonio se puede observar y desaparecer de la noche a la mañana y las empresas pueden decir hasta aquí llegamos; y además, dónde quedan las auditorías externas con dictámenes limpios o salvedades.

Cuando uno analiza esta problemática se piensa en la importancia que tiene el control interno a efectos de que pueda permitir que la empresa subsista y desarrolle. Como ejem- plo tenemos las quiebras del Banco de la República, Banco Nuevo Mundo, los grandes consorcios de empresas papeleras, empresas constructoras, etc.

Los que pagan por la deficiencia de control no son los accionistas porque generalmente ellos ya recuperaron su capital, quienes llevan la peor parte son los trabajadores y el Estado, porque las empresas al quebrar, también se llevan los tributos del IGV y la renta.

Ahora vamos a definir lo que es El Control Interno. Ése tiene como función primordial evaluar en forma independiente la eficiencia, eficacia, economía y equidad de las operaciones contables financieras, administrativas, de gestión de otra naturaleza de la entidad.

Es un proceso que lleva a cabo la gerencia de una organización y que debe estar diseñado para dar una seguridad razonable, en relación con el logro de los objetivos previamente establecidos en los siguientes aspectos básicos: 
El control interno es el encargado de craluar independientemente:

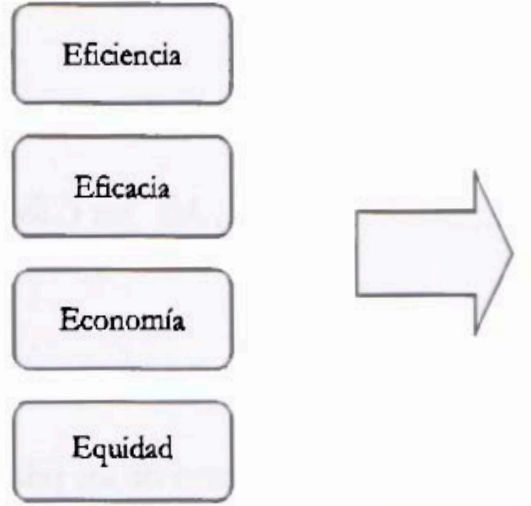

- Efectividad y eficiencia de las operaciones.

- Confiabilidad de los reportes financieros.

- Cumplimiento de leyes, normas y regulaciones aplicables.

\section{Objetivos del control interno}

Los objetivos del Control Interno deben lograr:

1. La obtención de la información financiera oportuna, confiable y suficiente como herramienta útil para la gestión y el control.

2. Promover la obtención de la información técnica y otro tipo de información no financiera para utilizarlas como elemento útil para la gestión y el control.

3. Procurar adecuadas medidas para la protección, uso y conservación de los recursos financieros, materiales, técnicos y cualquier otro recurso de propiedad de la entidad.

4. Promover la eficiencia organizacional de la entidad para el logro de sus objetivos y misión.

5. Asegurar que todas las acciones institucionales en la entidad se desarrollen en el marco de las normas constitucionales, legales y reglamentarias.

6. Idoneidad y eficiencia del recurso humano.

7. Crear conciencia de control.

\section{Elementos}

Los elementos del control interno son:

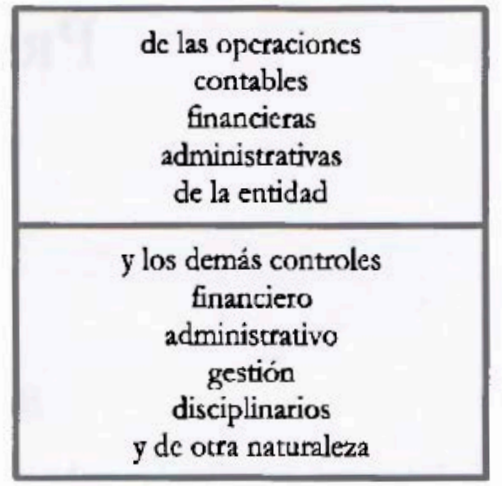

ALS

\section{Plan de organización}

Aún cuando no existe un solo concepto para definir organización, se entiende que ella se refiere a una estructura formalizada mediante la cual se identifican y grafican actividades y funciones, se determinan los cargos y las correspondientes líneas de autoridad, responsabilidad y coordinación. La organización proporciona el armazón que define las actividades que serán planeadas, ejecutadas, controladas y monitoreadas.

El organigrama describe la estructura formal de la entidad. Involucra por lo tanto:

- Determinación de funciones y actividades fundamentales para cumplir con los fines de la entidad.

- Integración de las funciones y actividades en unidades orgánicas.

- La jerarquización de la autoridad de manera que los grupos o individuos separados por la división de funciones en el trabajo actúen coordinadamente y enmarcados en claras líneas de responsabilidad.

- Identificación de áreas clave y líneas de información.

Un plan de organización adecuado se sustenta principalmente en lo siguiente:

- Independencia entre unidades operativas, sin que esto signifique ruptura de los canales de comunicación. 
- Reconocimiento de la necesidad de una efectiva segregación de funciones (operación, registro y custodia).

- Fijación de líneas de responsabilidad y delegación de autoridad apropiadas.

Algunos factores a considerar para evaluar el plan de organización institucional se resumen a continuación:

- La estructura organizacional debe ser lo más simple posible.

- Las responsabilidades funcionales deben segregarse con el fin de que una sola persona no controle todas las etapas relacionadas con una operación.

- Cada funcionario debe estar facultado para tomar decisiones y cumplir efectivamente con sus atribuciones con lo cual se evitan atrasos o inercia en la entidad.

- La responsabilidad de cada persona debe estar definida en forma precisa para que no pueda ser evadida o excedida en su ejercicio. La delimitación de responsabilidad permite evitar transferir las deficiencias incurridas a otras personas por inacción o acción inapropiada.

- Un funcionario que asigna funciones y delega autoridad a sus subordinados debe implementar mecanismos adecuados de control, con el fin de determinar si las tareas asignadas vienen cumpliéndose satisfactoriamente.

- Los empleados a quienes se les ha delegado autoridad están obligados a operar según los términos del encargo, debiendo consultar a su superior en casos de excepción.

- Toda persona está obligada a informar a su superior por la manera en que cumplió con sus tareas y por los resultados obtenidos en relación con lo que debió lograrse.

- La entidad debe ser tan flexible como para permitir una adecuada sincronización con los cambios en su estructura organizacional, a consecuencia de cambios en los planes, políticas y objetivos de las operaciones.

- Los organigramas o manuales de funciones son muy útiles para la comprensión de la organización de una entidad, sus líneas de autoridad y asignación de funciones y responsabilidades; evitándose la duplicidad de funciones o el conflicto al asignarlas.

\section{Planeamiento de actividades}

El planeamiento de actividades se orienta a determinar las necesidades de la entidad en cuanto a recursos financieros y humanos.

Al elaborar sus planes y proyectos la entidad debe considerar los aspectos siguientes:

- Los fondos autorizados y las limitaciones y restricciones.

- La necesidad de llevar a cabo todos los programas y proyectos y operaciones, de acuerdo a criterios de eficiencia y economía.

- La necesidad de cautelar que todos los recursos asignados sean utilizados en forma correcta y para los fines para los que fueron autorizados.

\section{Política}

Se puede definir política como la declaración general que guía el pensamiento durante la toma de decisiones. La política es una línea de conducta predeterminada que se aplica en una entidad para llevar a cabo todas las actividades, incluyendo aquellas no previstas. La política puede clasificarse en:

\section{- Política general}

Se establece para todas las entidades y para un caso específico. Generalmente se origina fuera de la entidad, como las leyes y los reglamentos directivas de sistemas administrativos.

\section{- Política específica}

Es escablecida por las altas direcciones de cada entidad y afectan a ésta en su totalidad. 


\section{- Politica para unidades}

Son aquellas establecidas en operatividad. Los niveles más bajos y su aplicación está limitada a las unidades operativas dentro de una misma entidad.

Los siguientes criterios son aplicables a la política de cada entidad:

- Establecer la política por escrito y organizarla en forma sistemática en guías o manuales, según los casos, para permitir efectuar las actividades autorizadas en forma efectiva, eficiente y económica, así como proporcionar seguridad razonable de que los recursos se encuentran debidamente protegidos.

- Comunicar la política a todos los funcionarios y empleados de la entidad cuyas obligaciones contribuyan al logro de sus objetivos.

- Elabora la políica en concordancia con la normatividad correspondiente y ser coherente con la política general dictada.

- Revisar la política en forma periódica debido al cambio de circunstancias, cuando se considere necesario.

\section{Procedimientos operativos}

Son los métodos utilizados para efectuar las actividades de acuerdo con las políticas establecidas. También son series cronológicas de acciones requeridas, guías para la acción que detallan la forma exacta en que deben realizarse ciertas actividades. Existe relación directa entre los procedimientos y las políticas. Una política aplicada por toda entidad es conceder a su personal vacaciones, los procedimientos establecidos por el Área de Personal para poner en práctica esa política deben permitir programar las vacaciones para evitar interrupciones en el ritmo de trabajo y llevar registros apropiados para asegurar que cada empleado disfrute de sus vacaciones.
Los mismos criterios a considerar para el establecimiento de la política de la entidad son aplicables a los procedimientos:

- Para promover la eficiencia y economía en las operaciones, los procedimientos aprobados deben ser simples y del menor costo posible. Para las operaciones que no son mecánicas en su ejecución, los procedimientos deben permitir el uso del criterio en situaciones fuera de lo común.

- Para reducir la posibilidad de errores e irregularidades, los procedimientos deben estar coordinados de manera que el trabajo realizado por un empleado sea revisado por otro, en forma independiente de sus propias obligaciones funcionales.

- Debe existir un programa adecuado de revisión periódica y mejora continua de los procedimientos aprobados.

\section{Personal}

La operatividad del sistema de control interno no depende exclusivamente del diseño apropiado del plan de organización, políricas y procedimientos, sino también de la selección de funcionarios con habilidad y experiencia y de empleados capaces de poder ejecutar sin dificultad los procedimientos establecidos por la administración.

Los elementos a considerar en el control del personal son:

\section{- Entrenamiento continuo}

Cuanto mejores sean los programas de capacitación, más apto será el personal. Esto permitirá la identificación clara de las funciones y responsabilidades de cada empleado y reducirá la ineficiencia y el desperdicio.

\section{- Eficiencia}

Después de la capacitación, la eficiencia dependerá del juicio personal aplicado a cada actividad. El interés de la administración por medir y alentar la eficiencia 
constituyen una forma de contribuir al logro de los objetivos del control interno.

\section{- Integridad y ética}

Constituye una de las columnas principales en que descansa la estructura del control interno.

\section{- Retribución}

Es un factor importante a considerar, dado que el personal que es compensado adecuadamente está dispuesto a lograr los objetivos dela entidad con entusiasmo y concentrar su atención en prestar con más eficiencia sus funciones y responsabilidades.

\section{Sistema contable e información financiera}

El sistema contable está constituido por los métodos y registros establecidos por la entidad para identificar, reunir, analizar, clasificar, registrar e informar sobre las transacciones realizadas en un período determinado. El sistema contable es un elemento importante del control financiero institucional al proporcionar la información financiera necesaria a fin de evaluar razonablemente las operaciones ejecutadas.

La presentación de reportes internos en toda entidad es necesaria para brindar a los funcionarios responsables una información confiable y actual sobre lo que está ocurriendo en realidad en cuanto al avance y progreso en el logro de los objetivos y metas establecidas. Tal información constituye la base fundamental del control gerencial en cualquier entidad.

Para presentar información a los niveles de decisión de la entidad deben considerarse, entre otros, los criterios siguientes:

- Los reportes deben elaborarse en forma simple y estar relacionados con la naturaleza del asunto, no debe incluirse información irrelevante para conocimiento de los funcionarios que lo soliciten como soporte para la toma de decisiones.

- La información financiera debe elaborarse de acuerdo con las atribuciones y responsabilidades de los funcionarios.

- Los funcionarios y empleados deben solamente reportar sobre asuntos de su exclusividad, competencia y funcional.

- Debe comprenderse el costo/beneficio resultante de la recopilación de datos y la elaboración de reportes con valor significativo.

Cuando sea posible, los reportes de avance y desempeño deben mostrar comparaciones referidas a:

- Normas establecidas sobre costos, cantidad y logros obtenidos.

- Partidas presupuestarias autorizadas y ejecutadas.

- Información sobre el desempeño en el periodo anterior.

\section{Sistema de control interno}

El sistema de control interno es el conjunto de todos los elementos en donde lo principal son las personas, los sistemas de información, la supervisión y los procedimientos.

El sistema de control interno es de vital importancia, ya que promueve la eficiencia, asegura la efectividad, previene que se violen las normas y los principios contables de general aceptación. Los directivos de las organizaciones deben crear un ambiente de control, un conjunto de procedimientos de control directo y las limitaciones del control interno.

La NIA 400 en el numeral 8 nos indica que el sistema del control interno significa que todas las políticas y procedimientos (controles internos) adoptados por la gerencia de 
- Aprobación y control de documentos.

- Comparación de datos internos con información de fuentes externas.

- Comparación de los recursos de los resultados de los arqueos de efectivo y valores y la toma de inventario con los registros contables.

- Limitar el acceso físico directivo de los activos y registros.

- Comparación y análisis de los estados financieros con los montos presupuestados.

Los procedimientos para mantener un buen control interno son:

- Delimitación de responsabilidades.

- Delimitación de autorizaciones generales y específicas.

- Segregación de funciones de carácter incompatible.

- Prácticas sanas en el desarrollo del ejercicio.

- División del procesamiento de cada transacción.

- Selección de funcionarios idóneos, hábiles, capaces y de moralidad.

- Rotación de deberes.

- Pólizas.

- Instrucciones por escrito.

- Cuentas de control.

- Evaluación de sistemas computarizados.

- Documentos prenumerados.

- Evitar uso de efectivo.

- Uso mínimo de cuentas bancarias.

- Depósito inmediato e intacto de fondos.

- Orden y aseo.

- Identificación de puntos clave de control en cada actividad, proceso o ciclo.

- Gráficas de control.

- Inspecciones e inventarios físicos frecuentes.

- Actualización de medidas de seguridad.

- Registro adecuado de toda la información.

- Conservación de documentos.

- Uso de indicadores.
- Prácticas de autocontrol.

- Definición de metas y objetivos claros.

- Hacer que el personal sepa por qué hace las cosas.

Algunos procedimientos de control interno en una empresa son:

- Arqueos periódicos de caja para verificar que las transacciones hechas sean las correctas.

- Control de asistencia de los trabajadores.

- $\mathrm{Al}$ adquirir responsabilidad con terceros, éstas se hagan solamente por personas autorizadas, teniendo también un fundamento lógico.

- Delimitar funciones y responsabilidades en todos los estamentos de la entidad.

- Hacer un conteo físico de los activos que en realidad existen en la empresa y cotejarlos con los que están registrados en los libros de contabilidad.

- Analizar si las personas que realizan trabajos dentro y fuera de la compañía los realizan adecuadamente y de una manera eficaz.

- Tener una numeración de los comprobantes de contabilidad en forma consecutiva y de fácil manejo para las personas encargadas de obtener información de estos.

- Controlar el acceso de personas no autorizadas a los diferentes departamentos de la empresa.

- Verificar que se están cumpliendo con todas las normas tributarias, fiscales y civiles.

- Analizar si los rendimientos financieros e inversiones hechas están dando los resultados esperados.

\section{Limitaciones inherentes del control interno}

Los sistemas del control interno y contable no pueden proporcionar a la gerencia evidencias concluyentes de que los objetivos son 


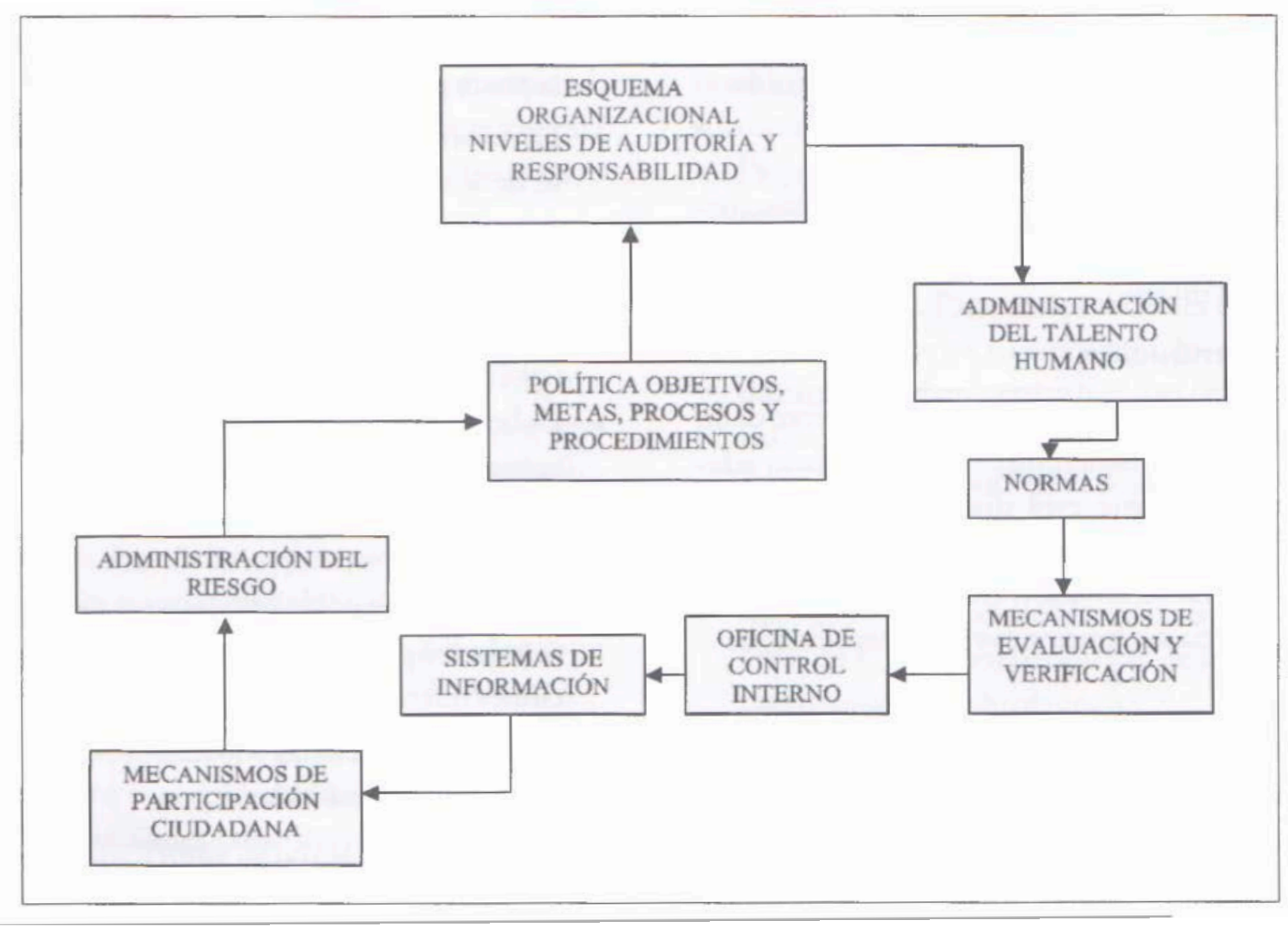

una entidad para el logro de los objetivos de la gerencia deben asegurar, hasta donde sea factible, la ordenada y eficiente conducción de su negocio, incluyendo la adherencia a las políticas de la gerencia, la salvaguarda de activos, la prevención y determinación de fraudes y errores, la exactitud e integridad de los registros contables y la oportuna preparación de información financiera confiable. El sistema de control interno se extiende más allá de aquellos aspectos que se relacionan directamente con las funciones del sistema contable.

En esta misma NIA, en el numeral 7, sistema contable significa la serie de tareas y registros de una entidad por medio de las cuales las transacciones son procesadas con el propósito de mantener registros financieros. Dicho sistema identifica, compila, analiza, calcula, clasifica, registra, sumariza y reporta transacciones y otros eventos.

\section{Procedimientos del control interno}

El procedimiento del control interno en la NIA 400, en el numeral 8 letra b), define como procedimiento de control «a aquellas políticas y procedimientos adicionales al ambiente de control que la gerencia ha establecido para lograr los objetivos específicos de la entidad». Los procedimientos específicos de control incluyen:

- Reporte, revisión y aprobación de conciliaciones.

- Revisión de la exactitud aritmética de los registros.

- Control de las aplicaciones y el ambiente del sistema de información computarizado, por ejemplo, para establecer controles sobre:

- Cambio a los programas de cómputo.

- Acceso a los archivos de dato.

- Mantener y revisar las cuentas de control y balance de comprobación. 
alcanzados debido a sus limitaciones inherentes. Tales limitaciones incluyen:

- Requerimiento anual de la gerencia de que el costo de implantación de un control interno no exceda los beneficios que se esperan de él.

- Muchos de los controles internos tienden más a ser dirigidos a transacciones rutinarias que a transacciones no rutinarias.

- El potencial de ocurrencia de error humano debido a negligencia, distracción, errores de juicio y no entendimiento de instrucciones.

- La posibilidad de eludir el control interno a través de la colusión de un miembro de la gerencia o de un empleado con terceros fuera o dentro de la empresa.

- La posibilidad de que una persona responsable de ejercer un control interno pueda abusar de esa responsabilidad, por ejemplo: un miembro de la gerencia eludiendo un control interno.

- La posibilidad que los procedimientos puedan ser inapropiados debido a cambios en las condiciones ya que el cumplimiento con los procedimientos pueden deteriorarse.

\section{CONCLUSIONES}

Considerando la importancia del control Interno en el manejo empresarial y el conocimiento de las personas para conseguir la optimización en los resultados del manejo de las operaciones es necesario:

- Definir el rol que juega el Control Interno en las empresas.

- La capacitación del personal para que no vulneren las normas de Control Interno.

- Mantener siempre una adecuada delimitación de funciones a través de un organigrama funcional y que guarde coherencia con el Manual de Funciones.

- Que el flujograma de los documentos vaya en relación al proyecto de desarrollo em- presarial y el cumplimiento de las metas y objetivos.

\section{REFERENCIAS}

1. Instituto Norteamericano de Contadores Públicos. Estudio y Evaluación del Control Interno por el Auditor.

2. Mirtha Luz Tizza Ballenas. Normas Técnicas de Control Interno para Abastecimientos Escuela Nacional de Control.

3. Sistema de Control Interno de una Pyme. Informativo Caballero Bustamante Revista de Asesoría Especializada

4. Auditoría. Normas Técnicas, Control Interno, Planificación del Trabajo, Objeto y Procedimientos. Tomo I. Edición 1996 Didáctica Multimedia S. A.

5. Informe especial. La Auditoría como instrumento de Control Informativo Caballero Bustamante. Tomo II. Edición 1999.

6. Segunda Norma: Control Interno Evaluación del Control Interno Informativo Caballero Bustamante. Tomo I, Edición 1998.

7. Sistema de Contabilidad y de control Interno - Consultas y Soluciones. Informativo Caballero Bustamante. Tomo II. Edición 1997.

8. El Informe del Auditor Independiente Responsabilidad de la Gerencia - La Gestión Empresarial y su Relación con el Control Interno. Informativo Caballero Bustamante. Tomo I. Edición 1997.

9. Jesús María Peña Bermúdez. Control, auditoría y revisoría fiscal. Incluye contraloría y ética profesional.

10. Samuel Alberto Mantilla B. Control Interno de los Nuevos Instrumentos Financieros.

11. Abraham Perdomo Moreno. Fundamentos de Control Interno. 